\title{
On a Fractional Nonlinear Hyperbolic Equation Arising from Relative Theory
}

\author{
Zujin Zhang, ${ }^{1}$ Xiaofeng Wang, ${ }^{2}$ and Zheng-an Yao ${ }^{3}$ \\ ${ }^{1}$ School of Mathematics and Computer Science, Gannan Normal University, Ganzhou 341000, China \\ ${ }^{2}$ College of Mathematics, Guangzhou University, Guangzhou 510006, China \\ ${ }^{3}$ Department of Mathematics, Sun Yat-sen University, Guangzhou 510275, China
}

Correspondence should be addressed to Zujin Zhang; zhangzujin361@163.com

Received 26 July 2013; Revised 17 October 2013; Accepted 2 November 2013

Academic Editor: Bashir Ahmad

Copyright (C) 2013 Zujin Zhang et al. This is an open access article distributed under the Creative Commons Attribution License, which permits unrestricted use, distribution, and reproduction in any medium, provided the original work is properly cited.

We obtain the existence of a weak solution to a fractional nonlinear hyperbolic equation arising from relative theory by the Galerkin method. Its uniqueness is also discussed. Furthermore, we show the regularity of the obtained solution. In our proof, we use harmonic analysis techniques and compactness arguments.

\section{Introduction}

This paper is concerned with the following fractional partial differential equations in $\mathbb{T}^{n}=\mathbb{R}^{n} / \mathbb{Z}^{n}$ :

$$
\begin{gathered}
\frac{\partial^{2} u}{\partial t^{2}}-\Lambda^{2 \alpha} u+|u|^{\rho} u=f \\
u(x, 0)=u_{0}(x), \\
\frac{\partial u}{\partial t}(x, 0)=u_{1}(x),
\end{gathered}
$$

where $\Lambda=(-\Delta)^{1 / 2}$ is the square root of the Laplacian operator, $\alpha \in(0,1), \rho \in[0, \infty)$ are two real parameters, and $f(x, t)$ is given. Equations (1)-(3) play an important role in nuclear force and relativistic theory.

The fractional diffusion operator $\Lambda^{2 \alpha}$ is nonlocal except when $\alpha \in \mathbb{N}$, which means that $\Lambda^{2 \alpha} u(x)$ depends not only on $u(y)$ for $y$ near $x$, but on $u(y)$ for all $y \in \mathbb{R}^{n}$. Fractional differential equations, arising from mathematical physics such as viscoelasticity, electrochemistry, control theory, porous media, and electromagnetism, now attract the interests of many mathematicians; see [1-4] and references therein. In the past ten years, the quasi-geostrophic equation with fractional dissipation has been extensively studied; see Constantin et al. [5-9] and references therein. The relativistic equation shares some similar difficulties with the quasi-geostrophic equation. However, the equations studied in this paper are more complicated in that the fractional diffusion operator and the nonlinear term in (1)-(3) bring new difficulties in passing to the limits of the approximate solutions, and hence, new devices must be introduced to overcome these obstacles.

When $\alpha=1,(1)-(3)$ become the standard equations, which were intensively studied in the past century. The readers are referred to[10-12] for more details.

Interestingly enough, the parabolic version of (1)-(3) with convection corresponds to the Navier-Stokes equations with damping; see $[13,14]$.

We now collect the notations in this paper. The square root of the negative Laplacian $-\Delta, \Lambda$ is given by (in terms of Fourier series)

$$
(\widehat{\Lambda f})_{k}=|k| \widehat{f}_{k}, \quad k \in \mathbb{Z}^{n}
$$

where $\widehat{f}_{k}$ is the Fourier coefficients of $f$ :

$$
\widehat{f}_{k}=\frac{1}{(2 \pi)^{n}} \int_{\mathbb{T}^{n}} f(x) e^{-i k \cdot x} \mathrm{~d} x, \quad k \in \mathbb{Z}^{n} .
$$

More generally, $\Lambda^{2 \alpha} f$ for $\alpha \in \mathbb{R}$ can be defined as

$$
\left(\widehat{\Lambda^{2 \alpha} f}\right)_{k}=|k|^{2 \alpha} \widehat{f}_{k}, \quad k \in \mathbb{Z}^{n} .
$$


We will also invoke the notion of homogeneous Sobolev space $\dot{H}^{s}\left(\mathbb{T}^{n}\right)(s \in \mathbb{R})$, which comprises all tempered distributions $f$ on $\mathbb{R}^{n}$ such that

$$
\|f\|_{\dot{H}^{s}\left(\mathbb{T}^{n}\right)}=\left(\sum_{k \in \mathbb{Z}^{n} \backslash\{0\}}|k|^{2 s}\left|\widehat{u}_{k}\right|^{2}\right)^{1 / 2} .
$$

We also recall the meaning of by the weak (weakly ${ }^{*}$ ) convergence in $L^{p}\left(0, T ; L^{q}\left(\mathbb{T}^{n}\right)\right)$. Since the dual of $L^{r}\left(\mathbb{T}^{n}\right) \quad(1 \leq r<$ $\infty)$ is $L^{r /(r-1)}\left(\mathbb{T}^{n}\right)$ and the space $C^{\infty}\left(\mathbb{T}^{n}\right)$ is dense in $L^{r}\left(\mathbb{T}^{n}\right)$ (noticing the periodic boundary conditions), we have

$$
f_{k} \rightarrow f \text { in } L^{p}\left(0, T ; L^{q}\left(\mathbb{T}^{3}\right)\right)
$$

with $1 \leq p, q<+\infty$ if and only if

$$
\begin{array}{r}
\int_{0}^{T} \int_{\mathbb{T}^{n}} f_{k} \phi \mathrm{d} x \mathrm{~d} t \longrightarrow \int_{0}^{T} \int_{\mathbb{T}^{n}} f \phi \mathrm{d} x \mathrm{~d} t \\
\forall \phi \in C_{0}^{\infty}\left(0, T ; C^{\infty}\left(\mathbb{T}^{n}\right)\right), \\
f_{k} \rightarrow f \text { in } L^{p}\left(0, T ; L^{q}\left(\mathbb{T}^{3}\right)\right)
\end{array}
$$

with $p=+\infty$ or $q=+\infty$ if and only if (9) holds.

We now close this introduction by outlining the rest of this paper. In Section 2, we prove the existence of a weak solution to (1)-(3); see Theorem 4 . The uniqueness of such weak solutions is discussed in Section 3; see Theorem 5. Finally, a regularity result is obtained in Section 4; see Theorem 6.

\section{Existence of a Weak Solution}

First, let us recall the following two fundamental lemmas.

Lemma 1 (see [15]). Let $X$ be a Banach space, $f \in L^{p}(0, T ; X)$, and $\partial f / \partial t \in L^{p}(0, T ; X)(1 \leq p \leq \infty)$, then $f \in C([0, T] ; X)$.

Lemma 2 (see $[15])$. Let $\mathscr{D}$ be a bounded domain in $\mathbb{R}^{n} \times \mathbb{R}_{+}$, and $\left\{g_{k}\right\}_{k=1}^{\infty}, g$ belong to $L^{q}(\mathscr{D}) \quad(1<q<\infty)$ with

$$
\left\|g_{k}\right\|_{L^{q}(\mathscr{D})} \leq C, \quad g_{k} \longrightarrow g \text { a.e. } \mathscr{D} \text {. }
$$

Then $g_{k} \rightarrow g$ in $L^{q}(\mathscr{D})$ weakly.

Let us now give the weak formulation of (1)-(3).

Definition 3. Let $T>0, u_{0} \in \dot{H}^{\alpha}\left(\mathbb{T}^{n}\right) \cap L^{\rho+2}\left(\mathbb{T}^{n}\right), u_{1} \in L^{2}\left(\mathbb{T}^{n}\right)$, and $f \in L^{2}\left(0, T ; L^{2}\left(\mathbb{T}^{n}\right)\right)$. A measurable function $u(x, t)$ is said to be a weak solution on $[0, T]$ to $(1)-(3)$ if the following conditions hold:

(1) $u \in L^{\infty}\left(0, T ; \dot{H}^{\alpha}\left(\mathbb{T}^{n}\right) \cap L^{\rho+2}\left(\mathbb{T}^{n}\right)\right)$ and $\partial u / \partial t \quad \epsilon$ $L^{\infty}\left(0, T ; L^{2}\left(\mathbb{T}^{n}\right)\right)$

(2) (1) holds in the sense of distributions; that is,

$$
\begin{aligned}
\int_{0}^{T} & \int_{\mathbb{T}^{n}} \frac{\partial u}{\partial t} \cdot \frac{\partial \phi}{\partial t} \mathrm{~d} x \mathrm{~d} t-\int_{0}^{T} \int_{\mathbb{T}^{n}} \Lambda^{\alpha} u \cdot \Lambda^{\alpha} \phi \mathrm{d} x \mathrm{~d} t \\
& +\int_{0}^{T} \int_{\mathbb{T}^{n}}|u|^{\rho} u \phi \mathrm{d} x \mathrm{~d} t \\
= & \int_{\mathbb{T}^{n}} u_{1} \phi_{t} \mathrm{~d} x+\int_{0}^{T} \int_{\mathbb{T}^{n}} f \phi \mathrm{d} x \mathrm{~d} t,
\end{aligned}
$$

for each $\phi \in C^{\infty}\left(\mathbb{T}^{n} \times(0, T)\right)$;
(3) $u(x, 0)=u_{0}(x)$ a.e. in $\mathbb{T}^{n}$;

(4) $(\partial u / \partial t)(x, 0)=u_{1}(x)$ a.e. in $\mathbb{T}^{n}$.

To see how item (4) in Definition 3 makes sense, we rewrite (1) as

$$
\frac{\partial^{2} u}{\partial t^{2}}=f+\Lambda^{2 \alpha} u-|u|^{\rho} u
$$

Noticing that [16]

$$
\Lambda^{2 \alpha} \text { is continuous from } \dot{H}^{\alpha}\left(\mathbb{T}^{n}\right) \text { to } \dot{H}^{-\alpha}\left(\mathbb{T}^{n}\right) \text {, }
$$

we have

$$
\Lambda^{2 \alpha} u \in L^{\infty}\left(0, T ; \dot{H}^{-\alpha}\left(\mathbb{T}^{n}\right)\right) .
$$

Also, due to the fact that

$$
\left\||u|^{\rho} u\right\|_{L^{(\rho+2) /(\rho+1)}\left(\mathbb{T}^{n}\right)}=\|u\|_{L^{\rho+2}\left(\mathbb{T}^{n}\right)}^{\rho+1},
$$

we deduce from (13) that

$$
\begin{aligned}
\frac{\partial^{2} u}{\partial t^{2}} \in & L^{2}\left(0, T ; L^{2}\left(\mathbb{T}^{n}\right)\right) \\
& +L^{\infty}\left(0, T ; \dot{H}^{-\alpha}\left(\mathbb{T}^{n}\right)+L^{(\rho+2) /(\rho+1)}\left(\mathbb{T}^{n}\right)\right) .
\end{aligned}
$$

Thus

$$
\frac{\partial^{2} u}{\partial t^{2}} \in L^{2}\left(0, T ; \dot{H}^{-\alpha}\left(\mathbb{T}^{n}\right)+L^{(\rho+2) /(\rho+1)}\left(\mathbb{T}^{n}\right)\right) .
$$

Hence, by item (1) of Definition 3, (18), and Lemma 1, we gather that

$$
\frac{\partial u}{\partial t} \in C\left([0, T] ; \dot{H}^{-\alpha}\left(\mathbb{T}^{n}\right)+L^{(\rho+2) /(\rho+1)}\left(\mathbb{T}^{n}\right)\right) .
$$

From this, we see that item (4) of Definition 3 makes sense, as claimed.

Now, we state our existence results in the following theorem.

Theorem 4. Let $T>0$,

$$
\begin{gathered}
u_{0} \in \dot{H}^{\alpha}\left(\mathbb{T}^{n}\right) \bigcap L^{\rho+2}\left(\mathbb{T}^{n}\right), \\
u_{1} \in L^{2}\left(\mathbb{T}^{n}\right), \\
f \in L^{2}\left(0, T ; L^{2}\left(\mathbb{T}^{n}\right)\right) ;
\end{gathered}
$$

then there exists at least one weak solution $u$ on $[0, T]$ to (1)(3), taking $u_{0}, u_{1}$ as initial data.

Proof. We use Galerkin method to establish the existence of such a solution.

Step 1 (construction of approximate solution). Let $\left\{w_{i}\right\}_{i=1}^{\infty}$ be a dense and total basis in $\dot{H}^{\alpha}\left(\mathbb{T}^{n}\right) \cap L^{\rho+2}\left(\mathbb{T}^{n}\right)$, and consider the approximate solution which has the form

$$
u_{m}(t)=\sum_{i=1}^{m} c_{i m}(t) w_{i}
$$


where $c_{i m}$ satisfy the following ordinary differential system:

$$
\begin{array}{r}
\left(\frac{\partial^{2} u_{m}}{\partial t^{2}}, w_{j}\right)-\left(\Lambda^{2 \alpha} u_{m}, w_{j}\right)+\left(\left|u_{m}\right|^{\rho} u_{m}, w_{j}\right)=\left(f, w_{j}\right), \\
1 \leq j \leq m, \\
(24) \\
u_{m}(0)=u_{0 m}=\sum_{i=1}^{m} \alpha_{i m} w_{i} \longrightarrow u_{0} \text { in } \dot{H}^{\alpha}\left(\mathbb{T}^{n}\right) \bigcap L^{\rho+2}\left(\mathbb{T}^{n}\right), \\
\text { as } m \longrightarrow \infty, \quad(25) \\
\frac{\partial u_{m}}{\partial t}(0)=u_{1 m}=\sum_{i=1}^{m} \beta_{i m} w_{i} \longrightarrow u_{1} \text { in } L^{2}\left(\mathbb{T}^{n}\right), \\
\text { as } m \longrightarrow \infty .
\end{array}
$$

Here and hereafter, we denote $(u, v)=\int_{\mathbb{T}^{n}} u v d x$ for $u, v \in$ $L^{2}\left(\mathbb{T}^{n}\right)$.

The system (24)-(26) is nonsingular because $\left\{w_{i}\right\}$ are linear independent. Thus, we may apply standard theory of ordinary differential equations to obtain the existence of a local solution to $(24)-(26)$ on $\left[0, t_{m}\right]$, for some $t_{m}>0$. We will then, in the next step, establish some a priori estimates of the obtained solutions which will ensure that $t_{m}=T$.

Step 2 (a priori estimates). Taking the inner product of (24) with $c_{i m}^{\prime}(t)$ in $L^{2}\left(\mathbb{R}^{n}\right)$, we obtain

$$
\begin{aligned}
\frac{1}{2} \frac{d}{d t} & {\left[\left\|\frac{\partial u_{m}}{\partial t}(t)\right\|_{L^{2}\left(\mathbb{T}^{n}\right)}^{2}+\left(\Lambda^{\alpha} u_{m}(t), \Lambda^{\alpha} u_{m}(t)\right)\right] } \\
& +\frac{1}{\rho+2} \frac{d}{d t} \int_{\mathbb{T}^{n}}\left|u_{m}(x, t)\right|^{\rho+2} d x=\left(f(t), \frac{\partial u_{m}}{\partial t}(t)\right) .
\end{aligned}
$$

Integrating over $[0, t]$ and invoking Hölder inequality then yield

$$
\begin{gathered}
\frac{1}{2}\left[\left\|\frac{\partial u_{m}}{\partial t}(t)\right\|_{L^{2}\left(\mathbb{T}^{n}\right)}^{2}+\left\|\Lambda^{\alpha} u_{m}(t)\right\|_{L^{2}\left(\mathbb{T}^{n}\right)}^{2}\right] \\
+\frac{1}{\rho+2}\left\|u_{m}(t)\right\|_{L^{\rho+2}\left(\mathbb{T}^{n}\right)}^{\rho+2} \\
\leq \frac{1}{2}\left[\left\|\frac{\partial u_{m}}{\partial t}(0)\right\|_{L^{2}\left(\mathbb{T}^{n}\right)}^{2}+\left\|\Lambda^{\alpha} u_{m}(0)^{2}\right\|_{L^{2}\left(\mathbb{T}^{n}\right)}\right] \\
+\frac{1}{\rho+2}\left\|u_{m}(0)\right\|_{L^{++2}\left(\mathbb{T}^{n}\right)}^{\rho+2} \\
+\int_{0}^{t}\|f(\tau)\|_{L^{2}\left(\mathbb{T}^{n}\right)}\left\|\frac{\partial u_{m}}{\partial \tau}(\tau)\right\|_{L^{2}\left(\mathbb{T}^{n}\right)} d \tau, \\
\forall t \in[0, T] .
\end{gathered}
$$

Due to (20), (25), and (26), we have that $\left\|\left(\partial u_{m} / \partial t\right)(0)\right\|_{L^{2}\left(\mathbb{T}^{n}\right)}^{2}$, $\left\|u_{m}(0)\right\|^{2}$, and $\left\|u_{m}(0)\right\|_{L^{\rho+2}\left(\mathbb{T}^{n}\right)}^{\rho+2}$ are uniformly bounded. Thus, (28) becomes

$$
\begin{aligned}
\frac{1}{2}\left[\left\|\frac{\partial u_{m}}{\partial t}(t)\right\|_{L^{2}\left(\mathbb{T}^{n}\right)}^{2}+\left\|\Lambda^{\alpha} u_{m}(t)\right\|_{L^{2}\left(\mathbb{T}^{n}\right)}^{2}\right] \\
\quad+\frac{1}{\rho+2}\left\|u_{m}(t)\right\|_{L^{\rho^{+2}\left(\mathbb{T}^{n}\right)}}^{\rho+2} \\
\leq C+\frac{1}{2} \int_{0}^{t}\|f(\tau)\|_{L^{2}\left(\mathbb{T}^{n}\right)}^{2} d \tau \\
\quad+\frac{1}{2} \int_{0}^{t}\left\|\frac{\partial u_{m}}{\partial \tau}(\tau)\right\|_{L^{2}\left(\mathbb{T}^{n}\right)}^{2} d \tau, \quad \forall t \in[0, T] .
\end{aligned}
$$

Noticing that $f \in L^{2}\left(0, T ; L^{2}\left(\mathbb{T}^{n}\right)\right)$, we have

$$
\begin{aligned}
\frac{1}{2}[ & \left.\left\|\frac{\partial u_{m}}{\partial t}(t)\right\|_{L^{2}\left(\mathbb{T}^{n}\right)}^{2}+\left\|\Lambda^{\alpha} u_{m}(t)\right\|_{L^{2}\left(\mathbb{T}^{n}\right)}^{2}\right] \\
& +\frac{1}{\rho+2}\left\|u_{m}(t)\right\|_{L^{+2}\left(\mathbb{T}^{n}\right)}^{\rho+2} \\
\leq & \frac{1}{2} \int_{0}^{t}\left\|\frac{\partial u_{m}}{\partial \tau}(\tau)\right\|_{L^{2}\left(\mathbb{T}^{n}\right)}^{2} d \tau+C, \quad \forall t \in[0, T] .
\end{aligned}
$$

Applying Gronwall's inequality then yields

$$
\left\|\frac{\partial u_{m}}{\partial t}(t)\right\|_{L^{2}\left(\mathbb{T}^{n}\right)} \leq C
$$

and hence,

$$
\left\|u_{m}(t)\right\|_{L^{\rho+2}\left(\mathbb{T}^{n}\right)} \leq C, \quad\left\|\Lambda^{\alpha} u_{m}(t)\right\|_{L^{2}\left(\mathbb{T}^{n}\right)} \leq C .
$$

Step 3 (passage to limit $m \rightarrow \infty$ ).

By (31) and (32), we have, up to a subsequence, still denoted by $u_{m}$, that

$$
\begin{gathered}
u_{m} \rightarrow u \text { weakly } * \text { in } L^{\infty}\left(0, T ; \dot{H}^{\alpha}\left(\mathbb{T}^{n}\right) \bigcap L^{\rho+2}\left(\mathbb{T}^{n}\right)\right), \\
\text { as } m \longrightarrow \infty, \\
\frac{\partial u_{m}}{\partial t} \rightarrow \frac{\partial u}{\partial t} \text { weakly } * \text { in } L^{\infty}\left(0, T ; L^{2}\left(\mathbb{T}^{n}\right)\right), \quad \text { as } m \longrightarrow \infty,
\end{gathered}
$$

and also that $u_{m}$ are uniformly bounded in $L^{2}\left(0, T ; \dot{H}^{\alpha}\left(\mathbb{T}^{n}\right)\right)$. Thus by Lemma 1 , we find that

$$
\begin{array}{r}
u_{m} \longrightarrow u \text { strongly in } L^{2}\left(0, T ; L^{2}\left(\mathbb{T}^{n}\right)\right), \quad \text { a.e. } \mathbb{T}^{n}, \\
\text { as } m \longrightarrow \infty .
\end{array}
$$

Hence, there exists a function $w(x, t)$ such that

$$
\begin{aligned}
\left|u_{m}\right|^{\rho} & u_{m} \longrightarrow w(x, t) \text { weakly } * \text { in } L^{\infty} \\
& \times\left(0, T ; L^{(\rho+2) /(\rho+1)}\left(\mathbb{T}^{n}\right)\right), \quad \text { as } m \longrightarrow \infty .
\end{aligned}
$$


By Lemma 2 and the fact that

$$
\left|u_{m}\right|^{\rho} u_{m} \longrightarrow|u|^{\rho} u, \quad \text { a.e. in } \mathbb{T}^{n},
$$

we know then that

$$
w=|u|^{\rho} u .
$$

Fixing $j$, we now pass to the limit $m \rightarrow \infty$ in (24) to deduce that

$$
\begin{aligned}
& \left(\frac{\partial^{2} u}{\partial t^{2}}(t), w_{j}\right)+\left(\Lambda^{2 \alpha} u(t), w_{j}\right) \\
& \quad+\left(|u(t)|^{\rho} u(t), w_{j}\right)=\left(f(t), w_{j}\right) .
\end{aligned}
$$

A simple density argument then shows that

$$
\left(\frac{\partial^{2} u}{\partial t^{2}}(t), v\right)+\left(\Lambda^{2 \alpha} u(t), v\right)+\left(|u(t)|^{\rho} u(t), v\right)=(f(t), v)
$$

for all $v \in \dot{H}^{\alpha}\left(\mathbb{T}^{n}\right) \cap L^{\rho+2}\left(\mathbb{T}^{n}\right)$.

Up to now, we have proved items (1) and (2) in Definition 3. Let us turn our attention to items (3) and (4) in Definition 3.

By (33) and Lemma 1 , we know $u_{m}(0) \rightarrow u(0)$ weakly in $L^{2}\left(\mathbb{T}^{n}\right)$, and from $(25), u_{m}(0) \rightarrow u_{0}$ in $\dot{H}^{\alpha}\left(\mathbb{T}^{n}\right) \cap L^{\rho+2}\left(\mathbb{T}^{n}\right)$; thus item (3) of Definition 3 is verified.

By (24) and (39), we see that

$$
\left(\frac{\partial^{2} u_{m}}{\partial t^{2}}(0), w_{j}\right) \longrightarrow\left(\frac{\partial^{2} u}{\partial t^{2}}(0), w_{j}\right) \text { weakly* }
$$

and by Lemma 1, we have

$$
\left(\frac{\partial u_{m}}{\partial t}(0), w_{j}\right) \longrightarrow\left(\frac{\partial u}{\partial t}(0), w_{j}\right)
$$

On the other hand, (26) implies that

$$
\left(\frac{\partial u_{m}}{\partial t}(0), w_{j}\right) \longrightarrow\left(u_{1}, w_{j}\right) .
$$

Hence

$$
\left(\frac{\partial u}{\partial t}(0), w_{j}\right)=\left(u_{1}, w_{j}\right), \quad \forall j \in \mathbb{N} .
$$

This verifies item (4) in Definition 3.

\section{Uniqueness of Weak Solutions}

In this section, we will discuss the uniqueness of weak solutions of (1)-(3). We only obtain partial results in case $0 \leq \rho \leq 2 \alpha /(n-2 \alpha)$. More precisely, we have the following theorem.

Theorem 5. Assuming as in Theorem 4, then there exists an unique weak solution of (1)-(3), in case $0 \leq \rho \leq 2 \alpha /(n-2 \alpha)$.
Proof. Let $u, v$ be two weak solutions for (1)-(3) given in Theorem 4 with the same datum. Then $w=u-v$ satisfies

$$
\frac{\partial^{2} w}{\partial t^{2}}+\Lambda^{2 \alpha} w=|v|^{\rho} v-|u|^{\rho} u
$$

$$
w(0)=0, \quad \frac{\partial w}{\partial t}(0)=0
$$

Also, we have

$$
\begin{gathered}
w \in L^{\infty}\left(0, T ; \dot{H}^{\alpha} \bigcap L^{\rho+2}\left(\mathbb{T}^{n}\right)\right), \\
\frac{\partial w}{\partial t} \in L^{\infty}\left(0, T ; L^{2}\left(\mathbb{T}^{n}\right)\right) .
\end{gathered}
$$

Taking the inner product of (44) with $\partial w / \partial t$ in $L^{2}\left(\mathbb{T}^{n}\right)$, we obtain

$$
\begin{array}{r}
\frac{1}{2} \frac{d}{d t}\left[\left\|\frac{\partial w}{\partial t}\right\|_{L^{2}\left(\mathbb{T}^{n}\right)}^{2}+\|w\|_{\dot{H}^{\alpha}\left(\mathbb{T}^{n}\right)}^{2}\right] \\
\quad=\int_{\mathbb{T}^{n}}\left(|v|^{\rho} v-|u|^{\rho} u\right) \frac{\partial w}{\partial t} d x .
\end{array}
$$

Invoking Hölder and Sobolev inequalities, we obtain

$$
\begin{aligned}
& \int_{\mathbb{T}^{n}}\left(|v|^{\rho} v-|u|^{\rho} u\right) \frac{\partial w}{\partial t} d x \\
& \leq(\rho+1) \int_{\mathbb{T}^{n}} \sup \left(|u|^{\rho},|v|^{\rho}\right)|w|\left|\frac{\partial w}{\partial t}\right| d x \\
& \leq C\left(\left\||u|^{\rho}\right\|_{L^{n / \alpha}\left(\mathbb{T}^{n}\right)}+\left\||v|^{\rho}\right\|_{L^{n / \alpha}\left(\mathbb{T}^{n}\right)}\right)\|w\|_{L^{q}\left(\mathbb{T}^{n}\right)}\left\|\frac{\partial w}{\partial t}\right\|_{L^{2}\left(\mathbb{T}^{n}\right)} \\
& \leq C\left(\|u\|_{\dot{H}^{\alpha}\left(\mathbb{T}^{n}\right)}^{\rho}+\|v\|_{\dot{H}^{\alpha}\left(\mathbb{T}^{n}\right)}^{\rho}\right)\|w\|_{L^{q}\left(\mathbb{T}^{n}\right)}\left\|\frac{\partial w}{\partial t}\right\|_{L^{2}\left(\mathbb{T}^{n}\right)} \\
& \leq C\left(\|u\|_{\dot{H}^{\alpha}\left(\mathbb{T}^{n}\right)}^{\rho}+\|v\|_{\dot{H}^{\alpha}\left(\mathbb{T}^{n}\right)}^{\rho}\right)\|w\|_{\dot{H}^{\alpha}\left(\mathbb{T}^{n}\right)}\left\|\frac{\partial w}{\partial t}\right\|_{L^{2}\left(\mathbb{T}^{n}\right)},
\end{aligned}
$$

where $(1 / q)+(\alpha / n)+(1 / 2)=1$, and we use $0 \leq \rho \leq 2 \alpha /(n-$ $2 \alpha)$.

Thus, (47) becomes

$$
\begin{aligned}
& \left\|\frac{\partial w}{\partial t}\right\|_{L^{2}\left(\mathbb{T}^{n}\right)}^{2}+\|w\|_{\dot{H}^{\alpha}\left(\mathbb{T}^{n}\right)}^{2} \\
& \quad \leq C \int_{0}^{t}\left(\left\|\frac{\partial w}{\partial t}\right\|_{L^{2}\left(\mathbb{T}^{n}\right)}^{2}+\|w\|_{\dot{H}^{\alpha}\left(\mathbb{T}^{n}\right)}^{2}\right) d \tau,
\end{aligned}
$$

and we get $w=0, u=v$ as desired.

\section{Regularity of the Weak Solution}

Now we discuss the regularity of solutions for (1)-(3). If the initial value and force are more regular, then so is the solution. 
Theorem 6. Let

$$
\begin{gathered}
u_{0} \in \dot{H}^{2 \alpha}\left(\mathbb{T}^{n}\right) \bigcap \dot{H}^{\alpha}\left(\mathbb{T}^{n}\right) \bigcap L^{\rho+2}\left(\mathbb{T}^{n}\right), \\
u_{1} \in \dot{H}^{\alpha}\left(\mathbb{T}^{n}\right) \bigcap L^{2}\left(\mathbb{T}^{n}\right), \\
f \in L^{\infty}\left(0, T ; L^{2}\left(\mathbb{T}^{n}\right)\right), \quad \frac{\partial f}{\partial t} \in L^{2}\left(0, T ; L^{2}\left(\mathbb{T}^{n}\right)\right),
\end{gathered}
$$

and $0 \leq \rho \leq 2 \alpha /(n-2 \alpha)$. Then there exists a unique weak solution for (1)-(3). Furthermore,

$$
\begin{aligned}
& u \in L^{\infty}\left(0, T ; \dot{H}^{2 \alpha}\left(\mathbb{T}^{n}\right)\right), \\
& \frac{\partial u}{\partial t} \in L^{\infty}\left(0, T ; \dot{H}^{\alpha}\left(\mathbb{T}^{n}\right)\right), \\
& \frac{\partial^{2} u}{\partial t^{2}} \in L^{\infty}\left(0, T ; L^{2}\left(\mathbb{T}^{n}\right)\right) .
\end{aligned}
$$

Proof. We just establish the a priori bounds, since the verification follows directly from passing to the limit for Galerkin approximate solutions.

Step 1 (bounds for initial data). Observing that

$$
0 \leq \rho \leq \frac{n}{n-2 \alpha} \Longrightarrow \frac{n}{2(\rho+1)} \geq-\alpha+\frac{n}{2},
$$

we see by Sobolev inequality that

$$
\left\|\left|u_{0}\right|^{\rho} u_{0}\right\|_{L^{2}\left(\mathbb{T}^{n}\right)} \leq\left\|u_{0}\right\|_{L^{2(\rho+1)}\left(\mathbb{T}^{n}\right)}^{\rho+1} \leq\left\|u_{0}\right\|_{H^{\alpha}\left(\mathbb{T}^{n}\right)^{n}}^{\rho+1} .
$$

By formula (13), we have

$$
\frac{\partial^{2} u}{\partial t^{2}}(0) \in L^{2}\left(\mathbb{T}^{n}\right)
$$

Step 2 (bounds for $\partial u / \partial t, \partial^{2} u / \partial^{2} t$ ). Differentiating (1) with respect to $t$, we find that

$$
\frac{\partial^{3} u}{\partial t^{3}}-\Lambda^{2 \alpha} \frac{\partial u}{\partial t}+(\rho+1)|u|^{\rho} \frac{\partial u}{\partial t}=\frac{\partial f}{\partial t} .
$$

Taking the inner product of (55) with $\partial^{2} u / \partial t^{2}$, we obtain by integration by parts that

$$
\begin{aligned}
\frac{1}{2} & \frac{d}{d t}\left[\left\|\frac{\partial^{2} u}{\partial t^{2}}\right\|_{L^{2}\left(\mathbb{T}^{n}\right)}^{2}+\left\|\Lambda^{\alpha} \frac{\partial u}{\partial t}\right\|_{L^{2}\left(\mathbb{T}^{n}\right)}^{2}\right] \\
\leq & \left(\frac{\partial f}{\partial t}, \frac{\partial^{2} u}{\partial t^{2}}\right)-(\rho+1)\left(|u|^{\rho} \frac{\partial u}{\partial t}, \frac{\partial^{2} u}{\partial t^{2}}\right) \\
& \equiv I_{1}+I_{2} .
\end{aligned}
$$

The first term $I_{1}$ can be easily dominated by using Hölder inequality as

$$
I_{1} \leq\left\|\frac{\partial f}{\partial t}\right\|_{L^{2}\left(\mathbb{T}^{n}\right)}\left\|\frac{\partial^{2} u}{\partial t^{2}}\right\|_{L^{2}\left(\mathbb{T}^{n}\right)} \leq \frac{1}{2}\left\|\frac{\partial f}{\partial t}\right\|_{L^{2}\left(\mathbb{T}^{n}\right)}^{2}+\frac{1}{2}\left\|\frac{\partial^{2} u}{\partial t^{2}}\right\|_{L^{2}\left(\mathbb{T}^{n}\right)}^{2} .
$$

To tackle $I_{2}$, we invoke Hölder and Sobolev inequalities to deduce that

$$
\begin{aligned}
I_{2} & \leq C\left\||u|^{\rho}\right\|_{L^{p}\left(\mathbb{T}^{n}\right)}\left\|\frac{\partial u}{\partial t}\right\|_{L^{q}\left(\mathbb{T}^{n}\right)}\left\|\frac{\partial^{2} u}{\partial t^{2}}\right\|_{L^{2}\left(\mathbb{T}^{n}\right)} \\
& \leq C\|u\|_{L^{\rho p}\left(\mathbb{T}^{n}\right)}^{\rho}\left\|\frac{\partial u}{\partial t}\right\|_{L^{q}\left(\mathbb{T}^{n}\right)}\left\|\frac{\partial^{2} u}{\partial t^{2}}\right\|_{L^{2}\left(\mathbb{T}^{n}\right)} \\
& \leq C\left\|\Lambda^{\alpha} u\right\|_{L^{2}\left(\mathbb{T}^{n}\right)}^{\rho}\left\|\Lambda^{\alpha} \frac{\partial u}{\partial t}\right\|_{L^{2}\left(\mathbb{T}^{n}\right)}\left\|\frac{\partial u}{\partial t}\right\|_{L^{q}\left(\mathbb{T}^{n}\right)} \\
& \leq C\left(\left\|\Lambda^{\alpha} \frac{\partial u}{\partial t}\right\|_{L^{2}\left(\mathbb{T}^{n}\right)}^{2}+\left\|\frac{\partial u}{\partial t}\right\|_{L^{q}\left(\mathbb{T}^{n}\right)}^{2}\right),
\end{aligned}
$$

where $p, q$ are chosen so that

$$
\frac{n}{\rho p}=-\alpha+\frac{n}{2}=\frac{n}{q},
$$

and thus

$$
\frac{1}{p}+\frac{1}{q}+\frac{1}{2} \leq 1
$$

Gathering (57) and (58) into (56), it follows that

$$
\begin{aligned}
& \frac{d}{d t}\left[\left\|\frac{\partial^{2} u}{\partial t^{2}}\right\|_{L^{2}\left(\mathbb{T}^{n}\right)}^{2}+\left\|\Lambda^{\alpha} \frac{\partial u}{\partial t}\right\|_{L^{2}\left(\mathbb{T}^{n}\right)}^{2}\right] \\
& \leq\left\|\frac{\partial f}{\partial t}\right\|_{L^{2}\left(\mathbb{T}^{n}\right)}^{2}+C\left(\left\|\Lambda^{\alpha} \frac{\partial u}{\partial t}\right\|_{L^{2}\left(\mathbb{T}^{n}\right)}^{2}+\left\|\frac{\partial u}{\partial t}\right\|_{L^{q}\left(\mathbb{T}^{n}\right)}^{2}\right) .
\end{aligned}
$$

Gronwall inequality then implies that

$$
\begin{gathered}
\left\|\frac{\partial^{2} u}{\partial t^{2}}\right\|_{L^{2}\left(\mathbb{T}^{n}\right)}^{2}+\left\|\Lambda^{\alpha} \frac{\partial u}{\partial t}\right\|_{L^{2}\left(\mathbb{T}^{n}\right)}^{2} \\
\leq e^{C t}\left[\left\|\frac{\partial^{2} u}{\partial t^{2}}(0)\right\|_{L^{2}\left(\mathbb{T}^{n}\right)}^{2}+\left\|\Lambda^{\alpha} u_{1}\right\|_{L^{2}\left(\mathbb{T}^{n}\right)}^{2}\right] \\
+e^{C t} \int_{0}^{t}\left\|\frac{\partial f}{\partial s}(s)\right\|_{L^{2}\left(\mathbb{T}^{n}\right)}^{2} d s<\infty .
\end{gathered}
$$

Step 3 (bounds for $u$ ). Rewriting (1) as

$$
\Lambda^{2 \alpha} u=\frac{\partial^{2} u}{\partial t^{2}}+|u|^{\rho} u-f
$$

we have $u \in L^{\infty}\left(0, T ; \dot{H}^{2 \alpha}\left(\mathbb{T}^{n}\right)\right)$, in view of similar inequalities satisfied by $|u|^{\rho} u$ as (53).

This completes the proof of Theorem 6 .

\section{Conflict of Interests}

The authors declare that there is no conflict of interests regarding the publication of this paper. 


\section{Acknowledgments}

Zujin Zhang was partially supported by the Youth Natural Science Foundation of Jiangxi Province (20132BAB211007), the Science Foundation of Jiangxi Provincial Department of Education (GJJ13658, GJJ13659), and the National Natural Science Foundation of China (11326138, 11361004). Xiaofeng Wang was partially supported by Guangzhou Higher Education Science and Technology Projection (2012A018).

\section{References}

[1] K. S. Miller and B. Ross, An Introduction to the Fractional Calculus and Differential Equations, John Wiley \& Sons, New York, NY, USA, 1993.

[2] I. Podlubny, Fractional Differential Equations, vol. 198 of Mathematics in Science and Engineering, Academic Press, San Diego, Calif, USA, 1999.

[3] J. Shatah and C. Zeng, "Schrödinger maps and anti-ferromagnetic chains," Communications in Mathematical Physics, vol. 262, no. 2, pp. 299-315, 2006.

[4] P. L. Sulem, C. Sulem, and C. Bardos, "On the continuous limit for a system of classical spins," Communications in Mathematical Physics, vol. 107, no. 3, pp. 431-454, 1986.

[5] P. Constantin, "Energy spectrum of quasigeostrophic turbulence," Physical Review Letters, vol. 89, no. 18, Article ID 184501, 2002.

[6] P. Constantin, "On the Euler equations of incompressible fluids," Bulletin of the American Mathematical Society, vol. 44, no. 4, pp. 603-621, 2007.

[7] P. Constantin, D. Cordoba, and J. Wu, "On the critical dissipative quasi-geostrophic equation," Indiana University Mathematics Journal, vol. 50, pp. 97-106, 2001.

[8] P. Constantin, A. J. Majda, and E. Tabak, "Formation of strong fronts in the 2-D quasigeostrophic thermal active scalar," Nonlinearity, vol. 7, no. 6, article 001, pp. 1495-1533, 1994.

[9] P. Constantin and J. Wu, "Behavior of solutions of 2D quasigeostrophic equations," SIAM Journal on Mathematical Analysis, vol. 30, no. 5, pp. 937-948, 1999.

[10] K. Jorgen, "Das anfangswert problem in grossen fur eine klasse nichllinearer wallengleichungen," Mathematische Zeitschrift, vol. 77, pp. 295-308, 1961.

[11] I. E. Segall, "Dispersion for nonlinear relativistic equations. II," Annales Scientifiques de l'École Normale Supérieure, vol. 1, no. 4, pp. 459-497, 1968.

[12] I. E. Segal, "The global Cauchy problem for a relativistic scalar field with power interaction," Bulletin de la Société Mathématique de France, vol. 91, pp. 129-135, 1963.

[13] X. J. Cai and Q. S. Jiu, "Weak and strong solutions for the incompressible Navier-Stokes equations with damping," Journal of Mathematical Analysis and Applications, vol. 343, no. 2, pp. 799-809, 2008.

[14] Z. J. Zhang, X. L. Wu, and M. Lu, "On the uniqueness of strong solution to the incompressible Navier-Stokes equations with damping," Journal of Mathematical Analysis and Applications, vol. 377, no. 1, pp. 414-419, 2011.

[15] J. L. Lions, Quelques Methodes de Resolution des Problems aux Limites Non Lineaire, Sun Yat-sen University Press, Guangzhou, China.
[16] E. M. Stein, Singular Integrals and Diferentiability Properties of Functions, Princeton University Press, Princeton, NJ, USA, 1986. 


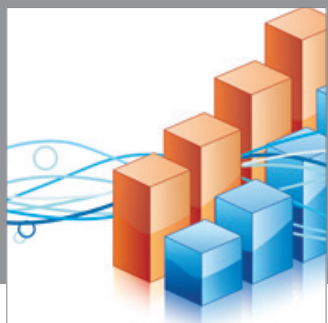

Advances in

Operations Research

mansans

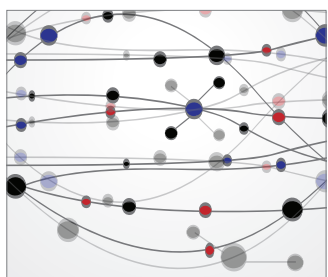

The Scientific World Journal
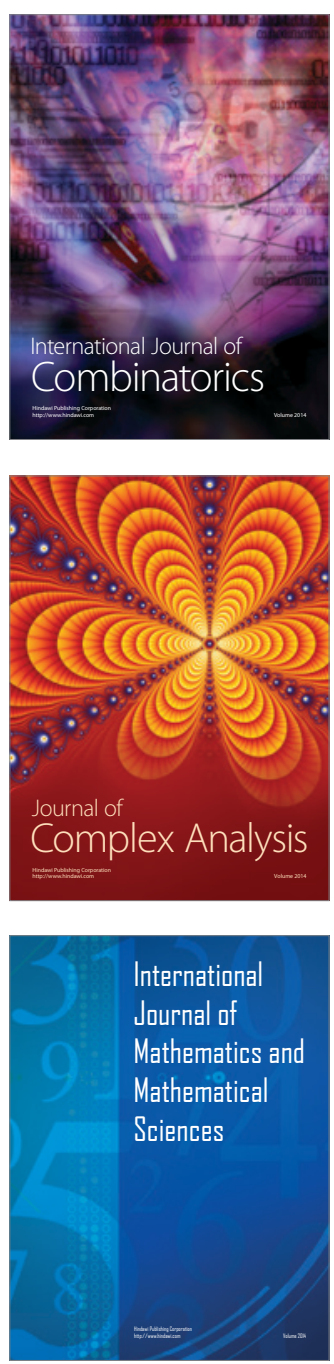
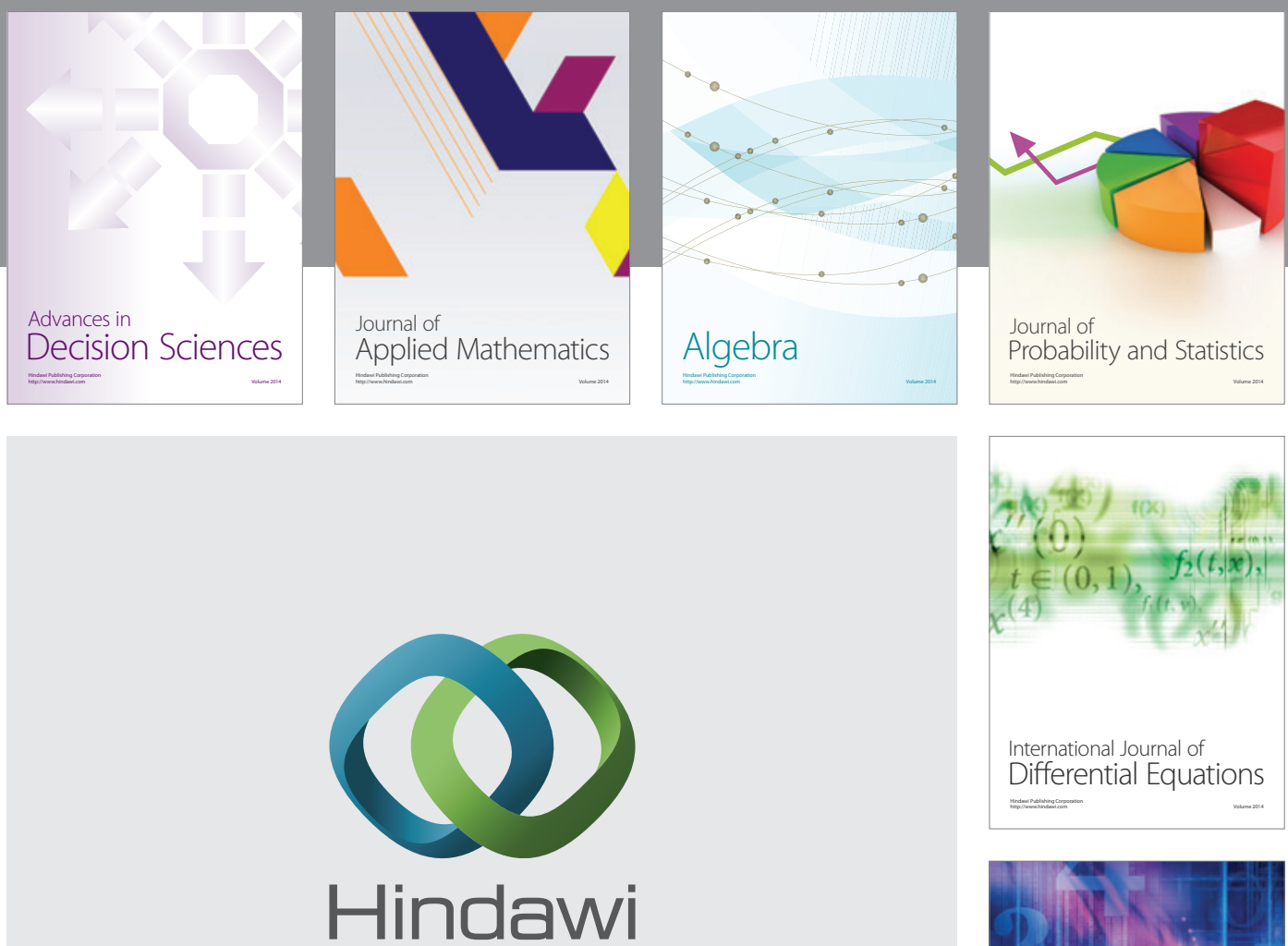

Submit your manuscripts at http://www.hindawi.com
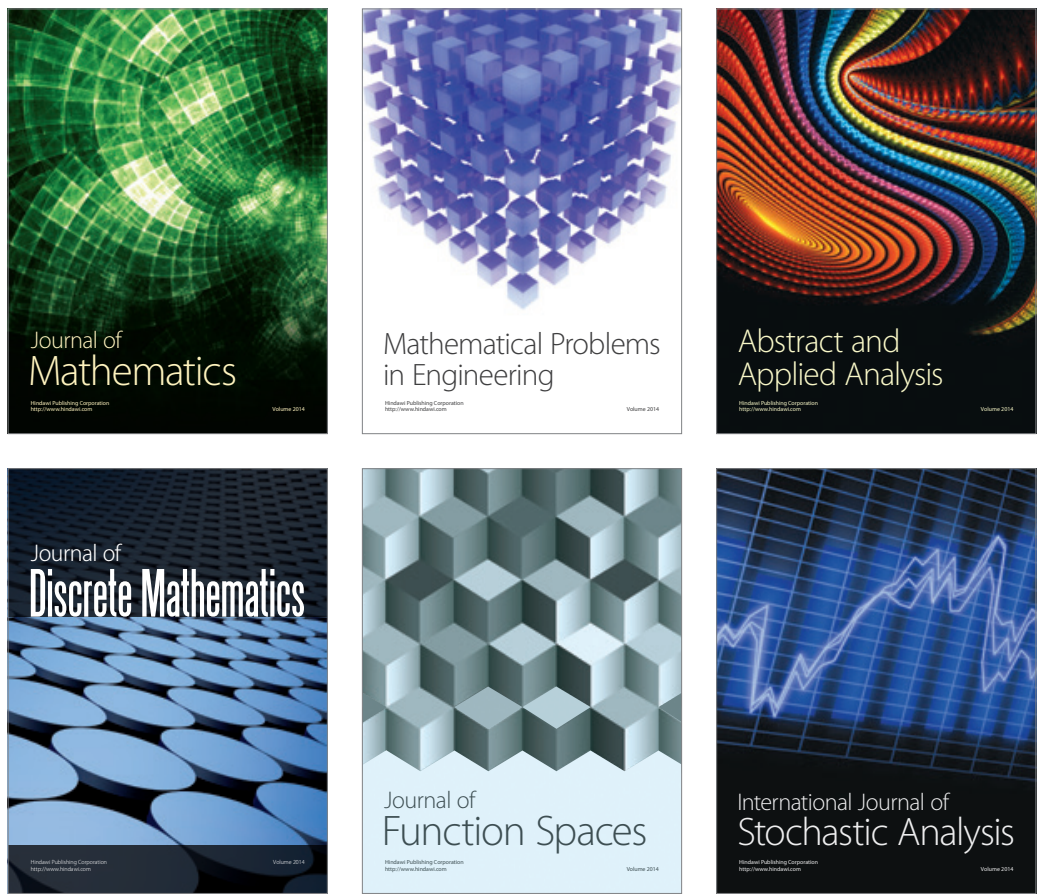

Journal of

Function Spaces

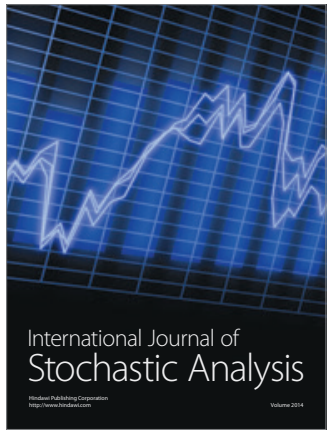

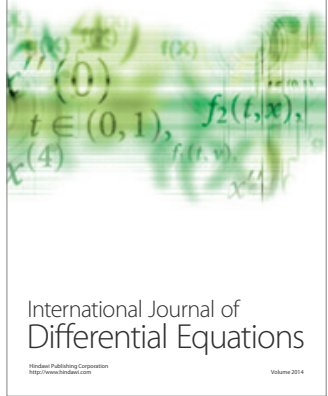
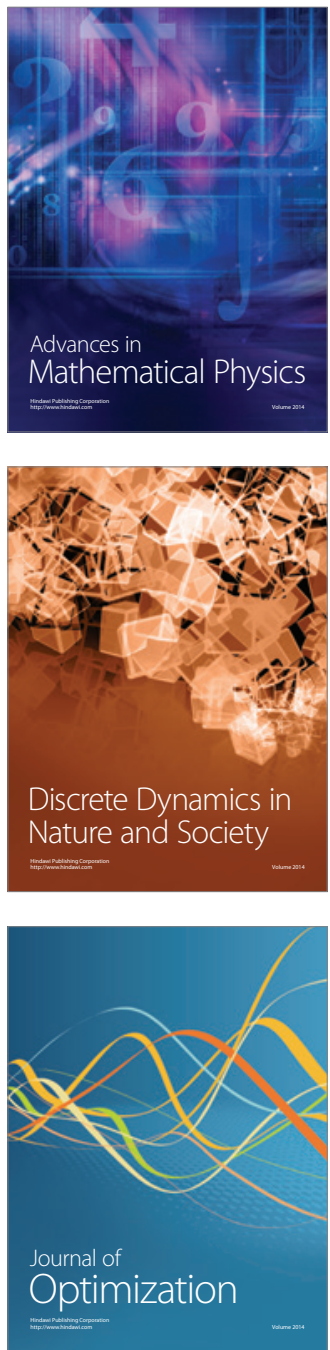